\title{
PERFIL DE FUNCIONALIDADE DE PACIENTES CRÍTICOS NOS PRIMEIROS DIAS DE VENTILAÇÃO MECÂNICA AVALIADA ATRAVÉS DA ESCALA PERME: UM ESTUDO TRANSVERSAL
}

\author{
Jessyka Cammilla Silva dos Santos ${ }^{1}$ \\ Helga Cecilia Muniz ${ }^{2}$ \\ Renata Moreira Zanetti ${ }^{3}$
}

\section{RESUMO}

Embora existam muitas ferramentas para avaliar a mobilidade funcional, nenhuma delas é considerada padrão-ouro para avaliação do paciente crítico. Para avaliar de forma rápida, específica e objetiva a funcionalidade do paciente crítico na Unidade de Terapia Intensiva (UTI) foi desenvolvida a Perme UCI Mobility Scale. O objetivo deste trabalho é analisar o perfil de mobilidade funcional dos pacientes críticos através da PERME nos primeiros dias de VMI. A pesquisa de campo, do tipo estudo clínico transversal descritivo, com o procedimento de coleta de dados, foi realizada no Hospital Getúlio Vargas (HGV) no período de agosto a novembro de 2019. Após a aprovação do comitê de ética e pesquisa, 71 pacientes foram avaliados. Os dados dos voluntários foram organizados em uma ficha de avaliação própria com a escala PERME e escore do Medical Research Council (MRC). Chegou-se a conclusão que o perfil da mobilidade funcional fica reduzido nos primeiros dias de VMI e a escala PERME é um instrumento válido na avaliação dos pacientes críticos.

Palavras-chave: Unidade de Terapia Intensiva. Debilidade muscular. Mecânica respiratória. Limitação de mobilidade. Respiração com pressão positiva.

Data de submissão: $20 / 06 / 2020$

Data de aprovação: 20/07/2020

\section{INTRODUÇÃO}

De acordo com o Ministério da Saúde (MS), em Janeiro de 2019 houve um total de 980.710 casos de internações nas emergências no Brasil. No nordeste, neste mesmo período, 263.836 pessoas foram internadas em emergências (DATASUS, 2019). Tais pacientes, também chamados de pacientes críticos, "são aqueles que possuem maior possibilidade de ir a óbito e/ou perder a função de sistemas e órgãos devido à debilidade de sua condição clínica" (BRASIL. Portaria n 2.338, 2011). A situação prévia desses pacientes quando associadas às complicações decorrentes do repouso prolongado no leito

1 Graduanda do curso de Fisioterapia da FMGR. E-mail: jessyka-cammilla@hotmail.com

2 Doutora em Fisioterapia pela UFPE e professora orientadora da FMGR. E-mail: helgamuniz@yahoo.com.br

3 Professora coorientadora da FMGR. E-mail: zanetti.rm@gmail.com 
e a ventilação mecânica (VM) podem ser agravadas. (SILVA et al., 2010; ARAÚJO, BORGES, 2010).

Uma prática comum no passado era acreditar que o paciente crítico não era capaz de resistir a qualquer tipo de atividade física. Acreditava-se que o repouso absoluto era a melhor maneira de tratamento para esses pacientes. Portanto, com os avanços tecnológicos, pesquisas, e com o incremento do conhecimento científico acerca do tema nas últimas decadas, foi comprovado que o repouso prolongado no leito é um fator colaborador para o retardo na recuperação desses pacientes (MUSSALEM et al., 2014; MORRIS et al., 2011).

Tal prática resultava na maior possibilidade de desenvolver lesão por pressão, osteoporose, pneumonia, atelectasia, déficit no sistema respiratório, tromboembolismo, fraqueza muscular, complicações hemodinâmicas e aumento do risco de infecção, além de poder causar contraturas, alterações das fibras musculares e afetar os barorreceptores que contribuem para a hipotensão postural e taquicardia, levando assim ao aumento do tempo de internação. Segundo alguns autores, esta prática colaborava para a piora do paciente, diminuição da qualidade de vida e aumento da mortalidade dentro de um ano após a alta da UTI. (LATRONICO, GOSSELINK, 2015; CARVALHO, BARROZO, 2014; MORRIS et al., 2011).

Ferramentas como Functional Independence Measure, Katz Index of Independence in Activities of Daily Living, Barthel Index, ICU Mobility Scale (IMS), Acute Care Index of Function, University of Rochester Acute Care Evaluation, Physical Function ICU Test (PFIT-s), a Chelsea Critical Care Physical Assesment Tool (CPA-x) e Functional Status Score for Intensive Care Unit (FSS -ICU) têm por objetivo avaliar a funcionalidade do paciente (PERME et al., 2014). Porém, nenhuma delas é considerada padrão-ouro na avaliação dos pacientes críticos em unidades de terapia intensiva (UTI) por não levarem em consideração as limitações dos pacientes, a mobilidade na UTI, a longa permanência na mesma, a presença de drenos, acessos e tubos que são barreiras de mobilidade ao paciente (KAWAGUCHI et al., 2016).

Por dispor da inserção de uma prótese na via aérea do paciente, que o liga ao ventilador e libera pressão positiva, a Ventilação Mecâniaca Invasiva (VMI) é considerada uma barreira de mobilidade. A interface pode ser um tubo orotraqueal, nasotraqueal ou cânula de traqueostomia. Embora seja muito usada nas UTIs, a VMI pode causar outros danos ao paciente, além da limitação da mobilidade, como: lesão traqueal, barotrauma, diminuição do débito cardíaco, toxicidade pelo uso do oxigênio, acúmulo de secreção por 
não ter uma tosse eficaz devido ao não fechamento da glote, déficit no transporte de muco por causa do tubo traqueal (o que contribui para possível hipoxemia), atelectasia e pneumonia. (MARTIN, 1994).

A VMI é indicada para pacientes que apresentam FR $>35 \mathrm{rpm}$, uso da musculatura acessória, $\mathrm{PaO} 2<60 \mathrm{mmHg}$ e/ou $\mathrm{PaCO} 2>55, \mathrm{pH}<7,25, \mathrm{SaO} 2<90 \%$ apesar do uso de $\mathrm{O} 2$ suplementar, rebaixamento no nível de consciência (Glasglow <8), obstrução das vias aéreas superiores, fadiga da musculatura acessória, instabilidade hemodinâmica, entre outros. (MACHADO et al., 2018).

Visando avaliar de forma rápida, específica e objetiva a mobilidade do paciente crítico na UTI, foi desenvolvido um instrumento confiável para padronizar esse tipo de avaliação - a Perme UCI Mobility Scale. Este instrumento avalia a capacidade do paciente em seguir os comandos verbais até a distância percorrida em 2 minutos (PERME et al., 2014). A escala tem sido utilizada para diversos estudos com pacientes submetidos a transplante hepático, transplante de pulmão e coração, substituição de válvula, reparo de aneurisma, insuficiência respiratória, sendo destinada a qualquer população de UTI e mostrando ser uma escala válida para avaliação (PEREIRA et al., 2019; PERME et al., 2014). Foi traduzida para a língua portuguesa em 2016 e caracterizou um alto grau de confiabilidade, mostrando assim que é um instrumento de grande credibilidade (ALMEIDA, 2018; KAWAGUCHI et al., 2016).

\section{METODOLOGIA}

Foi realizado um estudo do tipo transversal descritivo, sendo analisado o perfil da mobilidade funcional dos pacientes críticos nos primeiros dias de ventilação mecânica. A coleta de dados foi realizada no Hospital Getúlio Vargas (HGV), no período de agost o a novembro de 2019, após a aprovação do comitê de ética e pesquisa. (CAAE: 80113717.9.0000.5192).

Foram incluídos no estudo os pacientes críticos internados na UTI e na Unidade de Pacientes Graves (UPG) do Hospital Getúlio Vargas sob VMI, de ambos os sex os, e sem limitação de idade. Foram excluídos aqueles voluntários que possuíam instabilidade hemodinâmica e clínica para realização da avaliação, recusa em participar da pesquisa, e/ou pacientes com mais de 22 dias em VMI.

Os dados dos voluntários foram avaliados através de uma ficha de avaliação própria (apêndice A), contendo os seguintes tópicos: nome, idade, sexo, 
diagnóstico/causa da intubação, o tempo de VMI, tipo de via aérea, modo ventilatório, medicação, classificação de força muscular pelo escore do Medical Research Council (MRC) e escore de mobilidade funcional pela escala PERME.

O MRC consiste na avaliação de força em três grupos musculares para cada um dos membros superiores e inferiores (GREET et al., 2011). Os grupos musculares avaliados são: abdutores do ombro, flexores de cotovelo, extensores de punho, flexores do quadril, extensores do joelho e dorsiflexores do tornozelo. Cada grupo muscular recebe uma pontuação de 0 a 5 , sendo $0=$ paralisia; 1 = contração visível sem movimento; 2 = movimento muscular ativo com a gravidade eliminada; $3=$ movimento muscular ativo contra a gravidade; 4 = movimento ativo contra a gravidade e resistência; e $5=$ força normal, totalizando 60 pontos (VANHOUTTE et al., 2012). O Paciente que apresentar uma pontuação $<48$ sugere fraqueza muscular e $<36$, fraqueza grave (GREET et al., 2011).

A escala PERME UCI Mobility é dividida em 7 domínios que foram criadas para mostrar as dificuldades de mobilidade dos pacientes na UTI, sendo elas: estado mental, potenciais barreiras de mobilidade, força funcional, mobilidade no leito, transferências, marcha e endurance. Essas categorias são agrupadas em 15 itens e seu escore varia de 0 a 32 pontos (anexo A). Tal pontuação, quanto mais alta, indica uma melhor mobilidade; sendo baixa, indica que o paciente precisa de assistência, não havendo ponte de corte (KAWAGUCHI et al., 2016).

Os dados coletatos foram tabelados no programa Excel, sendo os dados quantitativos expressos em média e desvio-padrão, e os dados qualitativos expressos em frequência relativa.

\section{RESULTADOS}

Foram avaliados 71 indivíduos, tendo a média de idade de $62,78 \pm 19,39$ anos e o predomínio de pacientes do sexo masculino. O perfil diagnóstico de maior prevalência dos pacientes são os diagnósticos clínicos, uma vez que a maior parte deles passou menos de sete dias em VMI. O tipo de via aérea com maior prevalência nos pacientes avaliados foi o tubo orotraqueal (TOT) que estava, em sua maioria, no modo de ventilação a pressão controlada (PCV). Em relação aos valores da escala PERME, observou-se que tais pacientes possuem mobilidade funcional reduzida. Notou-se que o MRC em 91,5\% dos pacientes foi 0 por não entenderem o teste, medicações que faziam uso e/ou por apresentarem fraqueza muscular importante (Tabela 1). 
TABELA 1 - Características clínicas e funcionais da amostra.

\begin{tabular}{|c|c|}
\hline \multicolumn{2}{|c|}{$\begin{array}{c}\text { VOLUNTÁRIOS - №71 } \\
\text { (Média } \pm \text { DP ou \%) }\end{array}$} \\
\hline IDADE (ANOS) & $62,78 \pm 19,39$ anos \\
\hline SEXO & Homens $=57,7 \%$ \\
\hline \multicolumn{2}{|l|}{ DIAGNÓSTICO } \\
\hline Vascular & $2,81 \%$ \\
\hline Clínico & $64,7 \%$ \\
\hline Cirúrgico & $26,7 \%$ \\
\hline Neurológico & $5,63 \%$ \\
\hline \multicolumn{2}{|c|}{ TEMPO DE VMI (DIAS) } \\
\hline$<7$ & $70,4 \%$ \\
\hline $7-15$ & $23,9 \%$ \\
\hline$>15$ & $5,63 \%$ \\
\hline \multicolumn{2}{|c|}{ TIPO DE VIA AÉREA } \\
\hline TOT & $95,7 \%$ \\
\hline TQT & $2,8 \%$ \\
\hline TNT & $1,4 \%$ \\
\hline \multicolumn{2}{|c|}{ MODO VENTILATÓRIO } \\
\hline PCV & $81,6 \%$ \\
\hline PSV & $16,9 \%$ \\
\hline PRVC & $1,4 \%$ \\
\hline \multicolumn{2}{|l|}{ MEDICAÇÃo } \\
\hline Sedação & $1,80 \%$ \\
\hline Droga Vasoativa & $0,59 \%$ \\
\hline \multicolumn{2}{|l|}{ Neuromuscular } \\
\hline \multicolumn{2}{|c|}{ PERME (DOMÍNIOS) } \\
\hline Estado Mental & $0,59 \%$ \\
\hline Potenciais & $0,01 \%$ \\
\hline \multicolumn{2}{|l|}{ Barreiras } \\
\hline \multicolumn{2}{|l|}{ Mobilidade } \\
\hline Força Funcional & $0,33 \%$ \\
\hline Mobilidade & $0,15 \%$ \\
\hline \multicolumn{2}{|l|}{ no Leito } \\
\hline Transferências & $0 \%$ \\
\hline Marcha & $0 \%$ \\
\hline Endurance & $0 \%$ \\
\hline \multicolumn{2}{|l|}{ PERME (PONTOS) } \\
\hline $0-1$ & $87,3 \%$ \\
\hline $2-7$ & $9,8 \%$ \\
\hline$>7$ & $2,8 \%$ \\
\hline MRC (PONTOS) & \\
\hline
\end{tabular}


$\begin{array}{rr}0 & 91,5 \% \\ >0 & 8,4 \%\end{array}$

Fonte: autoria própria.

Idade descrita em média e desvio padrão. Demais dados expressos em porcentagens.

Legenda: TOT - Tubo orotraqueal; TQT - Traqueostomia; TNT - Tubo nasotraqueal; PCV - Ventilação a pressão controlada; PSV - Ventilação com pressão de suporte; PRVC - Volume controlado com pressão regulada.

\section{DISCUSSÃO}

O presente estudo mostrou que a média de idade dos pacientes de ambos os sexos foi maior que 60 anos. Ao longo dos anos vem sendo observado um aumento no número de internações em indivíduos nessa faixa étaria e a maior parte das internações é de causa clínica, devido ao uso contínuo de medicamento, risco maior de descompensar, fragilidade decorrente da idade e outros fatores. (MOTTA, HANSEL, SILVA, 2010).

A maioria das admissões realizadas nas UTIs do HGV é de pacientes do sexo masculino, devendo levar em consideração para tal prevalência os aspectos socioculturais, pois em geral os homens procuram os serviços de saúde em menor proporção, o que leva a maiores danos, como diagnósticos tardios, tratamentos especializados e caros (SILVEIRA et al., 2013). Há correspondência com o estudo realizado por Rodriguez et al. (2015), em que os resultados demonstraram predominância do sexo masculino de $61,6 \%$ devido ao baixo interesse dos mesmos pela sua saúde. Tais internações são seguidas pelas causas cirúrgicas. Em outro estudo realizado por Vieira et al. (2019), o resultado obtido das causas de internamento foi de 74,2\% clínicas e 19,4\% cirúrgicas.

Em relação ao tempo de permanência na UTI, foi verificado que 70,4\% dos pacientes ficaram menos de 7 dias internados. Segundo Oliveira et al. (2010), por existir uma variação na literatura sobre o tempo de permanência na UTI, o sétimo dia é considerado ponto de corte para o tempo de permanência na UTI. Stricker, Rothen e Takala (2003), corroboraram este dado no seu estudo ao utilizar o sétimo dia para definir a permanência prolongada na UTI.

A maior parte dos pacientes avaliados estava fazendo uso do TOT. Melo et al. (2007), em seu estudo, relatou que a prótese ventilatória mais utilizada para conectar o paciente ao ventilador mecânico foi o TOT, visto que a maioria dos pacientes ficaram internados até uma semana. Por se tratar de pacientes críticos que necessitam manter a via aérea segura, permeável, e por precisar de controle de ventilação pulmonar, a 
intubação orotraqueal é indicada (MATSUMOTO, CARVALHO, 2007).

$\mathrm{O}$ modo ventilatório mais utilizado foi o modo assistido-controlado PCV, ventilação a pressão controlada. Neste modo, é determinada a pressão que se quer ventilar o paciente, mantendo a segurança necessária para não lesar os pulmões do mesmo (DAMASCENO et al., 2006). É utilizado em situações em que a mecânica respiratória do paciente está comprometida e permite assim o controle adequado das pressões alveolares e nas vias aéreas (BARBAS et al., 2014). Devido à variação do fluxo que pode ocorrer pelas alterações da complascência ou resistência do paciente, este modo ventilatório proporciona mais conforto ao paciente (GARNERO et al., 2012).

O uso de droga vasoativa, bloqueadores neuromusculares, sedação e imobilidade no leito fazem com que a fraqueza muscular seja uma complicação frequente nos pacientes críticos (SILVA et al., 2017; JONGHE et al., 2009; MONTERO et al., 2005). As interrupções diárias desses medicamentos permitem a realização de uma avaliação física e mental mais eficaz do paciente, podendo reduzir o tempo de internamento e em VMI; permitem que eles acordem mais rápido, levando-os a se movimentar e realizar atividades espontâneas mais cedo, podendo assim evitar a fraqueza muscular. Sendo assim, é um importante passo para a melhoria da qualidade de vida do paciente, pois o ajuda a devolver a capacidade de se comunicar e interagir (MORRIS et al., 2008; JONGHE et al., 2005; KRESS et al., 2000).

Diante dos resultados da escala PERME, foi observado que na maioria dos pacientes apenas era possível realizar a avaliação do primeiro domínio (estado mental), seguido de força funcinoal, mobilidade no leito e potenciais barreiras à mobilidade com pontuação muito baixa. Desta forma, a maioria dos pacientes possuía uma pontuação de até 1 na escala PERME, indicando maior necessidade de assistência para mobilidade e mostrando que há uma relação entre a mobilidade dos pacientes e o tempo de VMI, sendo a mobilidade nos primeiros dias muito prejudicada. Isso se deve às potenciais barreiras, à mobilidade e ao tipo de medicamento que os pacientes faziam uso (PEREIRA et al., 2019; PERME et al., 2014).

A pontuação do MRC na maioria dos pacientes foi de 0 , devido à medicação, tempo de VMI e pontuação baixa na escala PERME. A imobilização completa ou quase completa nos pacientes que estão na UTI em VMI é comum. De $25 \%$ a 50\% dos pacientes em UTI em VMI apresentam fraqueza muscular. Tal fraqueza pode gerar complicações graves como longa permanência na UTI, uso prolongado da VMI e desmame difícil (HERMANS et al., 2007; JONGHE et al., 2004). 
Assim, os pacientes críticos na UTI, nos primeiros dias de VMI, têm em sua realidade menos atividade muscular devido a sua condição clínica, uso de sedação e demais medicações. Somados com os efeitos da idade avançada, acarretam a perda de massa muscular intensa. Desta forma, identificar o perfil da mobilidade do paciente na UTI com a escala PERME permite reconhecer alterações funcionais e auxilia no manejo terapêutico eficaz, sendo necessários ainda novos estudos a respeito da temática.

\section{CONCLUSÃO}

Como o perfil da mobilidade dos pacientes críticos fica reduzido nos primeiros dias de VMI, é possível concluir que a PERME é um instrumento viável para avaliar a mobilidade funcional e, por meio dela, é possível ajudar a reconhecer alterações funcionais futuras, evitar complicações decorrentes do repouso prolongado no leito e os efeitos deletérios da VMI, auxiliando no manejo terapêutico adequado.

\section{REFERÊNCIAS}

ARAUJO, A. E. T.; BORGES, F. S. Atuação da Fisioterapia motora no sistema músculoesquelético e na independência funcional dos pacientes em UTI. Rev Bras Ter Intensiva. Brasília-DF; 2010.

ALMEIDA, N. F. Aplicação do Perme Intensive Care Unit Mobility Score e ICU Mobility Scale em pacientes submetidos à cirurgia cardíaca. Ribeirão Preto, 2018.

BASTOS, J. L. D.; DUQUIA, R. P. Um dos delineamentos mais empregados em epidemiologia: estudo transversal. Scientia Medica, Porto Alegre, v. 17, n. 4, p. 229-232, out./dez. 2007.

BRASIL. Ministério da Saúde. Portaria $\mathrm{n}^{\mathrm{o}}$ 2.338, 2011. Estabelece diretrizes e cria mecanismos para a implantação do componente Sala de Estabilização (SE) da Rede de Atenção às Urgências. Disponível em: http://bvsms.saude.gov.br/bvs/saudelegis/gm/2011/prt2338_03_10_2011.html. Acesso em: 29 maio 2019.

CARVALHO, C. R., JUNIOR, C. T., FRANCA, S. A., Ve ntilação mecânica: princípios, análise gráfica e modalidades ventilatórias. J Bras Pneumol. 2007;33(Supl 2):S 54-S 70

CARVALHO, M. P., BARROZO, A. F., Mobilização Precoce no paciente crítico internado em Unidade de Terapia Intensiva. Braz. J. Surg. Clin. Res. V.8,n.3. set/nov, 2014.

CUNHA, S. Ventilação mecânica: métodos convencionais. Rev. HUPE, Rio de Janeiro, 2013. 
DAMASCENOS, et al. Ventilação Mecânica no Brasil. Aspectos Epidemiológicos. Rev. Bras. de Terapia Intensiva. Vol. 18 n. 3, 2006.

DE JONGHE et al, Intensive care unit-acquired weakness: Risk factors and prevention. Crit Care Med, 2009. Vol. 37.

DE JONGHE, B.; BASTUJI-GARIN, S.; SHARSHAR, T. et al. Does ICU-acquired paresis lengthen weaning from mechanical ventilation? Intensive Care Med 2004; 30:1117- 1121.

DE JONGHE, B.; BASTUJI-GARIN, S.; FANGIO, P. et al. Sedation algorithm in critically ill patients without acute brain injury. Crit Care Med 2005; 33:120-127.

GARNERO, A. J. et al. Modos controlados por presión versus volumen en laventilación mecánica invasiva. Med Intensiva. 2013; 37:292-8.

GREET, H. Interobserver agreement of medical research council sum-score and handgrip strength in the intensive care unit; MUSCLE \& NERVE, Jan. 2012.

JONGHE, M. D. et al. Paresis Acquired in the Intensive Care Unit: A Prospective Multicenter Study. JAMA. 2002.

KAWAGUCHI, Y. M. F. et al. Perme Intensive Care Unit Mobility Score e ICU Mobility Scale: tradução e adaptação cultural para a língua portuguesa falada no Brasil. J Bras Pneumol. 2016; 42(6):429-434.

KRESS, J.; POHLMAN, A.; O'CONNOR, M. et al. Daily interruption of sedative infusions in critically ill patients undergoing mechanical ventilation. N Engl J Med 2000; 342:14711477.

LATRONICO, N.; GOSSELINK, R. Abordagem dirigida para o diagnóstico de fraqueza muscular grave na unidade de terapia intensiva. Rev Bras Ter Intensiva. 2015.

MACHADO, F. D. et al. Ventilação Mecânica: Como iniciar. [s.1], 2018.

MATSUMOTO, T.; DE CARVALHO, W. B. Tracheal intubation. J Pediatr. Rio de Janeiro, 2007.

MARTIN, J. T. Mechanical ventilation. N Eng J Med. April, 1994.

MINISTÉRIO DA SAUDE. DEPARTAMENTO DE INFORMATICA DO SUS DATASUD. Informações em Saúde, Epidemiológicas e Morbidade: Banco de dados. Disponivel em: http://tabnet.datasus.gov.br/cgi/tabcgi.exe?sih/cnv/niuf.def. Acesso em: 29 maio 2019.

MOTERO, et al. Effect of critical illness polyneuropathy on the withdrawal from mechanical ventilation and the length of stay in septic patients. Crit Care Med.Vol. 33, Spain, 2005.

MORRIS, P. E.; GOAD, A.; THOMPSON, C. et al. Early intensive care unit mobility 
therapy in the treatment of acute respiratory failure. Crit Care Med 2008; 36:2238-2243.

MOTTA, C., HANSEL, C.; SILVA, J. Perfil de internações de pessoas idosas em um hospital público. Revista Eletrônica De Enfermagem. Rio de Janeiro, 2010.

MORRIS, P. E. et al. Receiving Early Mobility During An ICU Admission Is A Predictor Of Improved Outcomes In Acute Respiratory Failure. Am J Med Sci. 2011 May ; 341(5): 373-377.

MUSSALEM, M. A. M. et al. Influência da mobilização precoce na força muscular periférica em pacientes na Unidade Coronariana. ASSOBRAFIR Ciência, v. 5, n. 1, p. 7788, 2014.

OLIVEIRA, et al. Fatores associados à maior mortalidade e tempo de internação prolongado em uma unidade de terapia intensiva de adultos. Rev Bras Ter Intensiva. 2010; 22(3):250-256. 2009.

PARENTE, A. S.; VIEIRA, M. C. A. Perfil de morbidade e custos hospitalares com idosos no estado de Pernambuco. Revista Kairós, 21(1), 71-91. São Paulo; 2018.

PEREIRA, C. S. et al. Escala Perme como preditor de funcionalidade e complicações após a alta da unidade de terapia intensiva em pacientes submetidos a transplante hepático. Rev Bras Ter Intensiva. Nov. 2019.

PERME, C.; NAWA, R. K.; WINKELMAN, C.; MASUD, F. A tool to assess mobility status in critically ill patients: the Perme Intensive Care Unit Mobility Score. Methodist Debakey Cardiovasc J. 2014;10(1):41-9.

RODRIGUEZ, A. H. et al. Epidemiological characteristics and causes of deaths in hospitalized patients under intensive care. Rev Bras Enferm. 2016; 69(2):210-4.

SILVEIRA, R. E. et al. Gastos relacionados a hospitalizações de idosos no Brasil: perspectivas de uma década. Gestão e Economia em saúde. Uberaba, MG; 2013.

SILVA, I. T.; OLIVEIRA, A. A. Efeitos da mobilização precoce em pacientes críticos internados em UTI. C\&D-Revista Eletrônica da Fainor, Vitória da Conquista, v.8, n.2, p. 41-50, jul./dez. 2015.

SILVA, A. P. P.; MAYNARD, K.; CRUZ, M. R. Efeitos da fisioterapia motora em pacientes críticos: revisão de literatura. Rev Bras Ter Intensiva. 2010.

STRICKER, K.; ROTHEN, H. U.; TAKALA, J. Resource use in the ICU: short- vs. longterm patients. Acta Anaesthesiol Scand. 2003 May;47(5):508-15.

TERENCE, A. C.; FILHO, E. E. Abordagem quantitativa, qualitativa e a utilização da pesquisa-ação nos estudos organizacionais. XXVI ENEGEP - Fortaleza, CE, Brasil, 2006.

VIEIRA, et al. Características de óbitos dos pacientes internados em uma unidade de 
terapia intensiva de hospital terciário. J. Health Biol Sci. 2019; 7(1):26-31. Fortaleza, Ceará, 2019. 
Apêndice A - Ficha de avaliação própria

FICHA DE AVALIAÇ̃̃O - COLETA TCC

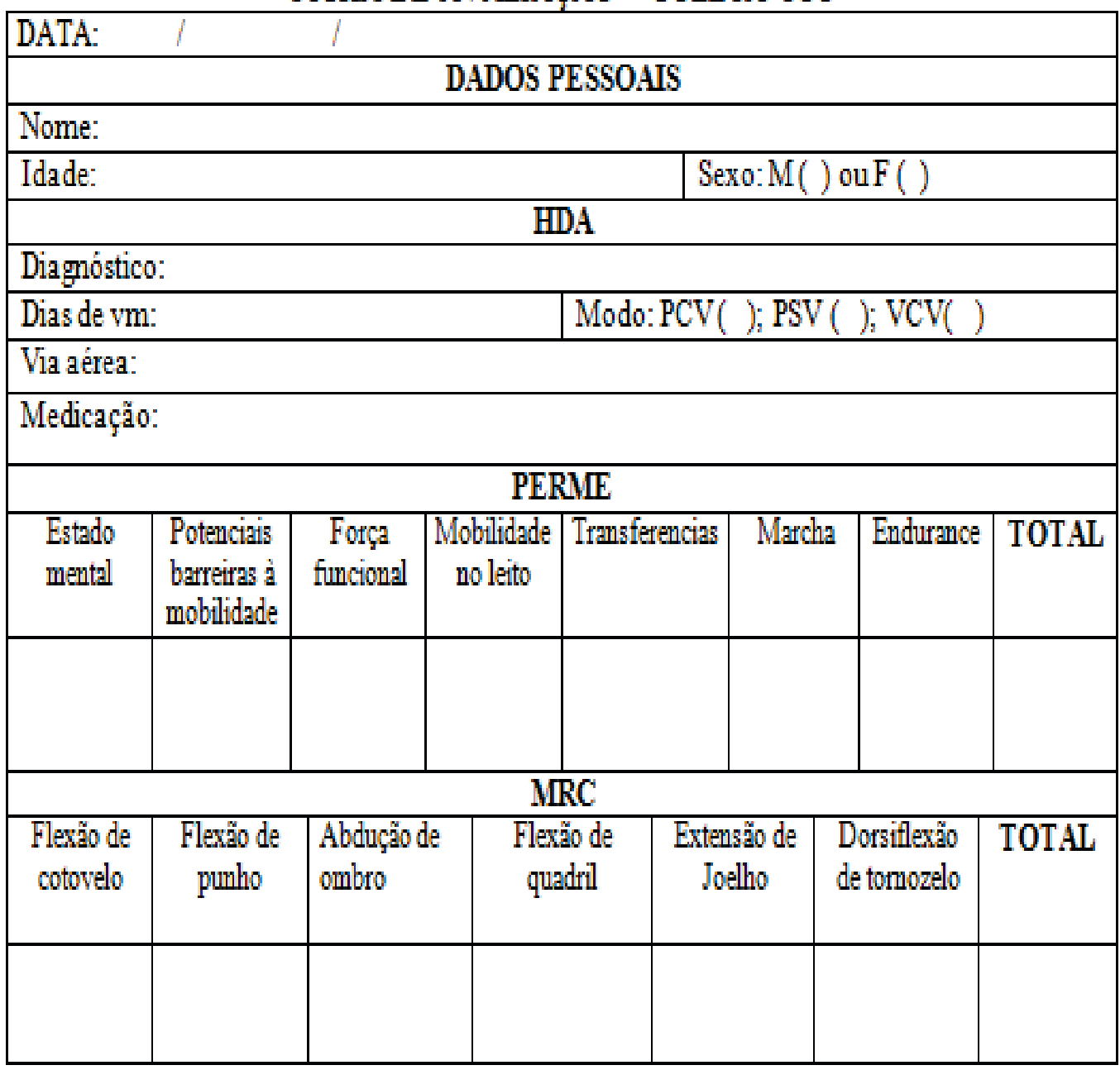

OBS: 


\section{Anexos A - Perme UCI Mobility Scale}

Quadro 3. Traduçăo do Escore de Mobilidade em UTI de Perme.A

\section{Nome do avaliador: Página 1}

Nome do paciente ou número:

ESTADO MENTAL

Pontuação máxima $=3$

Estado de alerta no começo da avaliação

Nāo responsivo 0

Letárgico $=1$

Acordado e alerta $=2$

0 paciente consegue seguir 2 entre 3 comandos?

Năo $=0$

$\operatorname{Sim}=1$

\section{POTENCIAIS BARREIRAS}

A MOBILIDADE

Pontuação máxima $=4$

- No mamento do contato

inicial com o paciente

ou a qualquer momento

durante as intervençōes

de mobilidade.

Pontuação máxima $=4$

MOBILIDADE NO LEITO

Pontuação máxima * 6

\section{Năo-Invasiva? •}

Sim $=0$

Năo $=1$

Dor ${ }^{*}$

Incapaz de determinar dor ou o paciente indica sentir dor $=0$

Sem dor $=1$

0 paciente apresenta 2 ou mais dos seguintes:" (circule)

de diálise, CCIP, SGP, SJP, sonda nasogástrica, dreno de tórax,

vácuo para feridas (VAC), ou outros.

$\operatorname{Sim}=0$

Nâo $=1$

O paciente está em infusão endovenosa? (infusão endovenosa

continua: vasopressores, inotrópicos, insulina, antiarritmicos,

sangue, etc)

$\operatorname{Sim}=0$

Nào $=1$ aproximadamente 20 graus, com o joetho estendido?

Nâo $=0$

$5 \mathrm{Sim}=1$ aproximadamente 45 graus, com o cotovelo estendido?

Năo $=0$

$\mathrm{Sim}=1$

$=1$

\section{Supino para sentado}

Năo avaliado OU Assistência total $(<25 \%)=0$

Dispositivos de oxigenoterapia, Cateter de Foley, TOT, Traqueostomia,

cateter central, cateter periférico, pressäo arterial invasiva, cateter

marcapasso temporário, cateter de artéria pulmonar, cateter epidural

(PCA), BIA, DAVE, TSRC, ventriculostomia, dreno lombar, curativo a

sedaçāo, antibióticos, fluidos, reposiçāo de eletrólitos, transfusāo de

Pernas - 0 paciente é capaz de erguer a perna contra a gravidade por Esquerdo Direito

Braços - 0 paciente é capaz de elevar o braço contra a gravidade por Esquerdo Direito

Máxima assistència $(25$ a $50 \%)=1$

Moderada assistência ( 50 a $75 \%$ ) $=2$

Minima assistência $(275 \%)$ OU Supervisão = 3

Equilibrio estảtico uma vez estabelecida a posição sentado à beira

do leito

Năo avaliado OU Assistência total $(<25 \%)=0$

Máxima assistência (25 a 50\%) $=1$

Moderada assistència $(50$ a $75 \%)=2$

\section{Pagina 2}

\section{MOBILIDADE NO LEITO}

Pontuaçāo máxima $=6$

\section{Supino para sentado}

Näo avaliado OU Assistência total $(<25 \%)=0$

Máxima assistência (25 a $50 \%)=1$

Moderada assistència (50 a 75\%) - 2

Minima assistência ( $>75 \%$ ) OU Supervisāo $=3$

Equilibrio estático uma vez estabelecida a posição sentado à beira

do leito

Năo avaliado OU Assistència total $(<25 \%)=0$

Máxima assistência $(25$ a 50\%) - 1

Moderada assistència ( 50 a $75 \%)=2$

Minima assistência ( $>75 \%)$ OU Supervisão -3 